FORMATION Formation emploi

Revue française de sciences sociales

100 | octobre-décembre 2007

De la formation professionnelle en Suisse

\title{
Formation continue dans les services publics en Suisse : quelles stratégies des acteurs dans un environnement managérialisé ?
}

Continuing vocational training in the Swiss civil service: personal strategies in a highly managerial environment

Weiterbildung im öffentlichen Dienst in der Schweiz: Welche Strategien haben die Betroffenen in einem Managementumfeld?

Yves Emery

\section{(2) OpenEdition}

Journals

Édition électronique

URL : http://journals.openedition.org/formationemploi/1274

DOI : 10.4000/formationemploi.1274

ISSN : 2107-0946

Éditeur

La Documentation française

Édition imprimée

Date de publication : 1 octobre 2007

Pagination : 79-91

ISSN : 0759-6340

Référence électronique

Yves Emery, «Formation continue dans les services publics en Suisse : quelles stratégies des acteurs dans un environnement managérialisé ? », Formation emploi [En ligne], 100 | octobre-décembre 2007, mis en ligne le 01 octobre 2009, consulté le 30 octobre 2020. URL : http://journals.openedition.org/ formationemploi/1274; DOI : https://doi.org/10.4000/formationemploi.1274 


\title{
DOSSIER
}

\section{Formation continue dans les services publics en Suisse: quelles stratégies des acteurs dans un environnement managérialisé ?}

Par Yves Emery*

\begin{abstract}
Le système où la carrière se développait surtout à l'ancienneté, sans réelle formation continue structurée, est remplacé par un système fortement organisé mais qui ne garantit plus la progression de carrière, ni même la reconnaissance des formations continues suivies.
\end{abstract}

Plus que jamais, la formation continue représente un enjeu essentiel dans l'environnement fortement évolutif au sein duquel administrations publiques et entreprises privées évoluent. Essentiel pour l'organisation, afin de l'aider à intégrer les nouvelles connaissances qui seront nécessaires à la mise en œuvre de ses programmes d'action, mais essentiel aussi pour le personnel, indépendamment de son niveau de qualification, pour maintenir sa motivation et son employabilité dans un marché de l'emploi de plus en plus compétitif. Aussi est-il surprenant de constater que la littérature sur la formation - en général et continue - revêt un caractère essentiellement prescriptif, attachant somme toute peu de considération à la manière dont les personnes concernées vivent concrètement leur formation continue, comment elles composent avec les objectifs et programmes de formation proposés ou imposés par leur employeur, et quelles stratégies elles mettent en œuvre pour poursuivre leurs propres objectifs. Cet article résume une recherche analysant la formation continue avant tout sous l'angle des acteurs concernés, permettant ainsi de mieux comprendre leurs logiques d'action. Conduite dans les services publics suisses, cette recherche apporte en outre un éclairage intéressant, intégrant les spécificités et contraintes de l'employeur public. Après avoir brièvement évoqué les particularités du système

* Yves Emery est docteur en sciences économiques de I'université de Genève, professeur à l'Institut de hautes études en administration publique (IDHEAP, Lausanne/ Suisse), responsable de la chaire de management public et gestion des ressources humaines. Directeur de recherches, il est consultant au sein de plusieurs services publics et auteur de nombreux ouvrages et articles scientifiques dont Paradoxes de la gestion publique, L'Harmattan, 2005, et Dynamiser les ressources humaines, PPUR, 2006 
public suisse et rappelé le design de la recherche, l'article résume, dans les deux paragraphes suivants, les résultats - tant quantitatifs que qualitatifs obtenus. Sur cette base, les principaux enseignements et conclusions font l'objet de la dernière partie de l'article, laquelle met en perspective les résultats obtenus pour questionner les tendances plus générales marquant la formation continue aux niveaux suisse et international.

\section{UN SYSTÈME FÉDÉRALISTE AVEC UNE LARGE AUTONOMIE DES ORGANISATIONS PUBLIQUES}

\section{Une fonction publique ouverte}

Fédéralisme oblige, le secteur public suisse se caractérise par une multitude d'organisations largement autonomes quant à leur mode de management et de gestion des ressources humaines. Le statut du personnel public, base légale définissant les conditions d'emploi des agents publics, prend le plus généralement la forme d'une loi votée par le Parlement. C'est dire que le Parlement fédéral pour l'administration fédérale, mais aussi les Parlements cantonaux pour les 26 administrations cantonales, et les quelques 2800 textes législatifs communaux sont en mesure de déterminer des conditions particulières d'emploi des fonctionnaires! D'où de très grandes différences dans la manière de gérer les employés publics (Emery et Ueberhart 1999), depuis la nature des rapports de travail (engagement unilatéral avec nomination des fonctionnaires, engagement contractuel de droit public, etc.), jusqu'aux modalités de rémunération (augmentations automatiques, en voie de disparition, rémunération tenant compte des performances des agents publics, en voie de généralisation), en passant naturellement par les questions de formation continue.

Contrairement au système de fonction publique dit «de carrière » (Germann 1996), en vigueur en France, où l'accès à la fonction publique est strictement réglementé et fait l'objet de concours d'entrée, la fonction publique suisse est dite « ouverte », c'està-dire que les candidat-e-s n'ont pas d'épreuve spéci- fique à réussir pour y accéder. Lorsqu'un poste est mis au concours, les autorités d'engagement (politiques, ou administratives, les chefs de service étant de plus en plus fréquemment désignés comme autorité d'engagement) déterminent les critères devant être remplis, des critères avant tout orientés vers les compétences techniques et managériales utiles dans le poste. À ces exigences peuvent s'ajouter des critères définis dans les statuts, mais ces derniers sont d'ordre administratif, tels que la détention d'un permis de travail ou encore le lieu de résidence. Toute personne estimant remplir ces critères peut postuler et être engagée, pour autant qu'elle parvienne à convaincre les autorités d'engagement de la qualité de sa candidature (lors d'entretiens de sélection avant tout). De même, aucune progression (ou «carrière ») n'est garantie, et souvent même annoncée aux agents publics; ces derniers doivent donc découvrir les opportunités professionnelles qui existent au sein de leur organisation, au gré des mises au concours découlant des postes devenus vacants.

Lévolution des effectifs de la fonction publique suisse est détaillée dans le tableau 1, où il apparâtt non seulement que les effectifs totaux sont en diminution, particulièrement au niveau de la Confédération, mais également que les effectifs principaux se trouvent aux niveaux cantonal et communal, expression du fédéralisme helvétique.

\section{Un environnement peu normé et très hétéroclite pour la formation continue}

La Suisse ne dispose d'aucune juridiction de portée générale qui contraindrait un employeur, public ou privé, à favoriser la formation continue, offrir un nombre de jours minimal annuel ou encore consacrer une partie de la masse salariale à la formation continue. De telles dispositions peuvent exister pour des catégories professionnelles spécifiques, par exemple les médecins, mais elles sont l'exception. Cela étant, chaque employeur peut décider, sur la base d'une politique du personnel spécifique et/ou d'une base réglementaire ad hoc, de favoriser, de rendre obligatoire ou encore d'offrir un droit à la formation continue, le plus souvent limité à quelques jours par an.

En revanche, fréquents sont les règlements qui précisent les modalités de soutien et de prise en 
Tableau 1

La fonction publique suisse.

Personnes actives occupées par niveau administratif, 1990-2000

\begin{tabular}{|l|c|c|c|c|c|c|}
\hline & \multicolumn{5}{|c|}{ Secteur public } & $\begin{array}{c}\text { Total des } \\
\text { actifs }\end{array}$ \\
\hline & Total & $\begin{array}{c}\text { Confédé- } \\
\text { ration }\end{array}$ & Cantons & Communes & $\begin{array}{c}\text { Autres } \\
\text { secteurs } \\
\text { publics (*) }\end{array}$ & $\begin{array}{c}\text { Secteur } \\
\text { public et } \\
\text { privé }\end{array}$ \\
\hline Total 2000 & 531558 & 80939 & 208488 & 186454 & 55677 & 3453796 \\
\hline Total 1990 & 599771 & 159890 & 220981 & 184399 & 34501 & 3580913 \\
\hline $\begin{array}{l}\text { Pour cent par rapport } \\
\text { au total des actifs 2000 }\end{array}$ & 15.4 & 2.3 & 6.0 & 5.4 & 1.6 & 100 \\
\hline Pour cent 1990 & 16.7 & 4.5 & 6.2 & 5.1 & 1.0 & 100 \\
\hline Variation 2000/1990 (N) & -68213 & -78951 & -12493 & 2055 & 21176 & -127117 \\
\hline Variation 2000/1990 (\%) & -11.4 & -49.4 & -5.7 & 1.1 & 61.4 & -4 \\
\hline $\begin{array}{l}\text { Répartition au sein } \\
\text { du secteur public 2000 }\end{array}$ & 100.0 & 15.2 & 39.2 & 35.1 & 10.5 & - \\
\hline $\begin{array}{l}\text { Répartition } \\
\text { secteur public 1990 (\%) }\end{array}$ & 100.0 & 26.7 & 36.8 & 30.7 & 5.8 & - \\
\hline
\end{tabular}

$\left(^{*}\right)$ : Comprend toutes les autres organisations qui, sans appartenir formellement à l'administration (fédérale, cantonale ou communale), prennent en charge des tâches publiques (par exemple, Établissements autonomes de droit public pour le contrôle des véhicules).

Note de lecture : ainsi, les effectifs au niveau des cantons ont diminué de plus de 12000 employés publics au cours de cette période de 10 ans, pour atteindre 208488 en 2000, alors que la proportion des effectifs cantonaux dans le total des emplois (actifs) baissait légèrement de 6.2 à $6 \%$.

Source : Bochsler, Koller, Sciarini, Traimond et Trippolini, 2004.

charge de la formation continue par l'employeur (public). Dès le moment où un agent public participe à une formation continue, la décharge temporelle qui lui est accordée, la prise en charge des frais directs et indirects de formation sont le plus souvent décrites dans le détail, sur la base de l'idée directrice suivante: plus la formation est directement utile dans le poste, et plus elle est suggérée par la hiérarchie plutôt que souhaitée par la personne concernée, plus la prise en charge est importante. Ainsi, une formation imposée, notamment suite à une évaluation de performances qui aurait mis en évidence certaines lacunes à combler chez l'agent public évalué, sera intégralement supportée par l'employeur. Dans ce contexte, il existe un foisonnement de pratiques au sein des organisations publiques en Suisse, qui rend toute étude comparative à grande échelle problématique.

\section{MANAGER PAR LES PERFORMANCES : L'IMPACT SUR LA FORMATION CONTINUE}

Nous avons souhaité approfondir sur une base plus qualitative, au moyen d'études de cas, les pratiques de formation existant au sein d'organisations publiques en transformation, sous l'impulsion de modes de gestion inspirés de la Nouvelle gestion publique $(\mathrm{NPG})^{1}$, mouvement ayant eu d'importantes réper-

\footnotetext{
${ }^{1}$ Mouvement datant d'une quinzaine d'années, consistant à repenser le fonctionnement de l'administration pour l'orienter davantage vers des objectifs à atteindre fixés par les autorités politiques, tout en lui accordant une marge de manœuvre accrue - notamment budgétaire - dans son fonctionnement. La NGP inspire fortement la LOLF (loi organique relative aux lois de finances du $1^{\text {er }}$ août 2001, qui s'applique entièrement pour la première fois au budget 2006) laquelle peut donner aux lecteurs français une compréhension plus directe du système.
} 
cussions dans notre pays (Emery 2000 ; Emery et Giauque, 2005). Contrairement à la plupart des études sur cette question, qui se focalisent sur des variables socioculturelles comme déterminants de la formation continue, nous avons cherché à identifier l'influence des pratiques de management internes sur les comportements de formation continue d'agents publics occupant des positions de cadre de premier niveau hiérarchique depuis la base.

La recherche visait à décrire et expliquer les comportements de formation continue des cadres dans les services publics, dans un contexte générateur de tensions, puis à tenter de reconstituer leurs stratégies personnelles pour faire face à ces tensions et poursuivre leurs objectifs personnels. Ces dernières années en effet, nombre d'organisations publiques ont développé la formation continue, en l'appuyant sur une politique du personnel qui insiste particulièrement sur le développement des compétences (Emery et Lambelet-Rossi, 2000) ; d'un autre côté, sous l'impulsion de la NGP, ces mêmes organisations introduisent des pratiques de management des performances qui génèrent une pression accrue dans la réalisation du travail et sont susceptibles d'entraver considérablement cette initiative de formation continue, notamment en rendant l'accès difficile du point de vue de la gestion du temps. Nous tentons ainsi de savoir comment les personnes réagissent à ces attentes potentiellement contradictoires.

Pour l'essentiel, la NGP introduit une manière différente de gérer l'action publique, davantage axée sur les outputs (résultats) individuels et collectifs, c'està-dire sur les objectifs fixés et les performances réalisées, plutôt que sur les inputs (moyens), soit l'attribution des ressources budgétaires et l'élaboration du cadre législatif (Emery 2006). Au niveau collectif, la NGP se traduit par l'élaboration de mandats de prestations au terme desquels les services administratifs doivent atteindre des objectifs de performance négociés avec l'autorité politique, qui serviront également de base à l'évaluation de leur efficacité - soit l'atteinte des objectifs fixés - et de leur efficience, soit l'utilisation rationnelle des moyens alloués. Au niveau individuel, en relation avec de nouvelles pratiques de gestion des ressources humaines qui conduisent à introduire notamment des entretiens périodiques d'évaluation, les agents publics sont de plus en plus gérés et évalués sur la base d'objectifs individuels (Emery et Gonin, 2006).

Dans ce contexte résolument axé sur la performance, quelles incidences ce nouveau cadre d'action exercet-il sur les agents publics dans le domaine de leur formation continue?

En fonction de diverses publications traitant de manière connexe notre sujet de recherche, nous avons postulé une corrélation négative entre l'intensité des pratiques de management des performances et le comportement actif de formation continue, à travers l'hypothèse principale suivante : les comportements « actifs » de formation continue sont liés à la nature du management appliqué dans le service : plus le management est axé sur les performances, moins le comportement de formation continue des salariés est actif.

Nous avons défini les comportements «actifs» de formation comme un sous-ensemble des comportements de formation continue, qui exclut la formation imposée par l'employeur et qui englobe donc les activités de formation continue soutenues par l'organisation, mais non-obligatoires, ainsi que les activités d'autoformation, pendant et hors du temps de travail. Cette approche est originale et nous verrons que la distinction opérée ici s'est avérée fondamentale dans la mise en valeur des données obtenues.

Nous avons opté pour une acception large de la notion de formation continue, soit toute action de formation intentionnelle, (formelle, informelle ou non formelle), entreprise en cours d'emploi par les personnes interrogées, qu'elle vise l'activité exercée ou le développement personnel. Les comportements de formation continue ont été identifiés sur la base d'un relevé systématique portant sur une année entière (2000), un relevé tant quantitatif (heures consacrées à la formation continue) que qualitatif, présentant le détail des activités réalisées.

Les services analysés représentent deux domaines d'activités typiques pour les administrations publiques : «administratif/fiscal» où trois services ont été sélectionnés, et «infrastructure/entretien» où trois autres services ont été retenus. Au sein de ces derniers, deux catégories bien distinctes de cadres ont été choisies : des cadres professionnels techniques venant de la base (contremaîtres), et des cadres 


\section{Encadre 1 \\ La méthode utilisée}

Une méthodologie mixte combinant les approches quantitative et qualitative a été mise en œuvre. Une analyse quantitative, portant notamment sur la variance des comportements actifs (en heures annuelles) selon les types d'environnements retenus et des variables individuelles de contrôle (par exemple, le niveau de formation antérieur). Une approche qualitative, qui s'inspire de l'analyse stratégique des acteurs, un cadre théorique reconnu dans le courant de la sociologie des organisations (Crozier et Friedberg 1977 ; Friedberg 1997). II s'agit d'une approche qui se focalise sur la véritable qualité d'acteur des membres de l'organisation, c'est-à-dire leur capacité, même réduite, à intervenir sur leur environnement. L'acteur, selon la définition de Crozier/Friedberg, est à comprendre comme le "sujet agissant», en l'occurrence la personne qui utilise de manière plus ou moins active les possibilités présentes dans son environnement pour atteindre ses objectifs de formation continue.

Les services retenus ont été décrits au moyen de caractéristiques visant à identifier de manière complète leur «processus formationnel », tel que perçu par les personnes rencontrées : existence d'une politique en matière de formation continue (FC), pratiques de FC (ressources offertes au personnel, essentiellement sous formes de cours, manifestations diverses, supports didactiques, ainsi que les règles d'accès à ces ressources), culture de FC (attitude de la direction et de la hiérarchie face à la FC).

Quant aux stratégies, elles ont été reconstituées en identifiant les principaux objectifs en matière de FC poursuivis par les acteurs interrogés (52 personnes), leurs ressources, atouts et contraintes, ainsi que la marge de manœuvre perçue qui en découle. Les entretiens menés, de type semi-directif, ont pris entre 45 minutes et deux heures. Les questions étaient orientées sur les activités de formation continue et les motivations sous-jacentes, permettant ensuite de reconstituer les stratégies d'acteurs mises en œuvre. Précisons encore que le jeu des acteurs, au sens de Crozier/Friedberg, n'a pas pu être reconstitué, étant donné que la hiérarchie directe et les collègues des personnes interviewées n'ont pas pu être rencontrés.

formés au sein de hautes écoles (ingénieurs), permettant une analyse plus fine. En fonction de l'hypothèse posée, ces six services sont différenciés selon leurs outils de management des performances (MP) : - MP+, pour les 2 services disposant d'un management des performances tant collectives (par exemple : contrat de prestations entre le politique et l'administration) qu'individuelles (par exemple: contrat individuel d'objectif);

- MP+/-, pour les 2 services ne disposant que d'un management des performances individuelles ;

- MP-, pour les 2 services « traditionnels » ne disposant d'aucun management des performances.

Le détail des démarches entreprises, analyses effectuées et recommandations figure dans l'ouvrage issu de la recherche (Emery, Budde et ChuardDelaly, 2004).

\section{DES DISPARITÉS INTER-INDIVIDUELLES INDÉPENDANTES DU MODE DE MANAGEMENT}

De l'exploitation des données détaillées fournies par les 52 cadres ayant participé à la recherche, il ressort que :

- la moyenne des heures totales de formation continue s'élève à près de 98 heures sur l'année 2000 (médiane à 30 heures), dont 66 heures sont consacrées à la formation continue active ; les cours et séminaires sont la forme la plus fréquente pour cette formation continue active, représentant 39,5 heures par année, alors que 2,5 heures sont consacrées à des conférences, foires, expositions et que 24 heures sont consacrées à la lecture, l'utilisation de didacticiels et la qualification expérientielle (apprendre en « faisant ») ; 
- sur 66 heures de formation continue active, 41 heures ont été soutenues financièrement par l'organisation et 33 heures ont pu être réalisées pendant le temps de travail ;

- 12 personnes ont déclaré n'avoir participé à aucune formation continue active et 6 uniquement à des formations continues obligatoires. Ces chiffres élevés, même s'ils ne sont pas représentatifs, ont de quoi surprendre dans l'environnement actuel.

L'hypothèse principale n'a pas pu être démontrée par l'analyse de variance. En effet, nous avons constaté une influence probable de la gestion des performances sur les activités de formation continue obligatoires, mais non pas sur les heures actives. Dans notre échantillon, une gestion des performances collectives et individuelles (MP+) s'accompagne d'un nombre d'heures et de cours de formation continue obligatoires significativement supérieur (statistiquement, avec les réserves liées à l'échantillon restreint) aux unités ne possédant aucune gestion des performances. Cette tendance ne se confirme en revanche pas pour les formations nonobligatoires, c'est-à-dire pour les comportements actifs de FC. C'est donc bien sur la formation obligatoire, et non sur la formation active comme nous le pensions initialement, que le management des performances exerce une influence directe et, qui plus est, positive.

Quant aux variables de contrôle individuelles retenues dans cette recherche, elles nous permettent de confirmer les tendances maintes fois démontrées sur le sujet: nous pouvons conclure que le niveau de formation est la variable qui influence le plus l'engagement des individus dans les activités de formation continue, que celles-ci soient volontaires (actives) ou obligatoires. Nous n'avons trouvé qu'une seule influence spécifique aux comportements actifs : il s'agit d'une corrélation avec la facette de personnalité «Ouverture aux valeurs » qui a été évaluée au moyen du questionnaire de personnalité NEO-PI-R $(\text { Costa, 1996 })^{2}$. Les personnes disposées à remettre en question les valeurs sociales, politiques et reli-

\footnotetext{
${ }^{2}$ L'un des questionnaires reconnus au plan international pour apprécier les différentes facettes de la personnalité, en tenant compte des traits principaux de la personnalité identifiés par la littérature spécialisée (approche dite «par les traits» ou encore des «big five »).
}

gieuses auraient davantage tendance à s'engager volontairement dans des activités de formation continue, constat qui mériterait bien entendu d'être approfondi par des recherches ultérieures. Cela dit, comment les cadres réagissent-ils et quelles stratégies mettent-ils en œuvre en matière de formation continue?

\section{DES STRATÉGIES D'ACTEURS FONDÉES SUR LE COURT TERME}

Nous résumons ici les éléments essentiels conduisant à l'élaboration des stratégies d'acteurs, soit les objectifs, ressources, atouts et limites composant la réalité de nos interlocuteurs, ainsi que la marge de manœuvre dont ils disposent.

Concernant tout d'abord les objectifs concrètement poursuivis par les personnes interrogées, relevons que si leur discours général plaide pour une FC importante et indispensable, ouvrant sur l'évolution dans tous les domaines, leurs objectifs réels demeurent très fortement focalisés sur l'utilité fonctionnelle à court terme et donc ancrés dans le domaine d'activité actuel, voire dans la fonction actuelle: ces objectifs sont essentiellement réactifs face à la contrainte d'un processus de plus en plus imposé ( $c f$. ci-dessus). Force est de constater la parfaite conformité de ces objectifs personnels avec les objectifs organisationnels et, partant, le caractère exceptionnel des objectifs de FC à moyen terme et proactifs. Le fait que les formations suivies ne sont souvent pas certifiantes $^{3}$, peut en partie expliquer ce constat. La notion d'employabilité, la vision à plus long terme, n'ont quasiment jamais été évoquées par nos interlocuteurs, si ce n'est comme objectifs abstraits liés à la FC. Rappelons encore que nous avons renoncé à déterminer les stratégies en matière de $\mathrm{FC}$ au niveau individuel, pour chacun de nos interlocuteurs, puisque cette démarche aurait nécessité, entres autres, des entretiens avec les principaux acteurs agissant dans l'environnement professionnel des

\footnotetext{
${ }^{3}$ Nombre de formations suivies ont été conçues spécifiquement pour un employeur, à sa demande, et ne mènent donc pas à un quelconque diplôme.
} 
personnes interrogées, démarche dépassant de loin nos ressources.

Les ressources en FC utilisées par nos interlocuteurs se résument pour l'essentiel à l'offre de FC qui leur est faite : elles sont avant tout localisées dans l'environnement de travail immédiat (collègues) et du service (programme proposé ou imposé); dans une moindre mesure au niveau de l'organisation (programme en circulation) et encore moins à l'extérieur. Les ressources les plus légitimes, donc celles qui bénéficient d'un soutien maximal (financier et temporel) de la part de l'employeur, sont celles qui sont le plus intégrées au service et dont l'utilisation correspond le mieux au critère de l'utilité fonctionnelle immédiate.

Les principaux atouts susceptibles d'être utilisés par nos interlocuteurs pour faire valoir leurs objectifs en matière de FC sont :

- des atouts liés à la personne, comme l'investissement professionnel (« en vouloir») et personnel (souplesse, y compris en dehors du temps de travail) et les capacités de négociation; l'ancienneté et l'habitude de recourir à la formation continue peuvent être invoquées pour favoriser, mais également pour éviter de suivre de la FC ;

- des atouts liés à la fonction exercée, notamment l'autonomie d'action et la légitimité accrues du fait des responsabilités assumées en tant que cadre, et qui se répercutent sur les possibilités de FC;

- des atouts liés au réseau de relations, hiérarchiques et transversales, puisqu'il peut s'agir de bien s'entendre avec sa hiérarchie de proximité - susceptible de bloquer les demandes de FC - mais également de faire valoir des liens tissés dans d'autres secteurs de l'organisation pour légitimer des demandes supplémentaires et obtenir des informations sur les opportunités.

Les limites et contraintes avec lesquelles nos interlocuteurs doivent composer et qui s'observent le plus fréquemment sont les suivantes :

- les conditions de production et de réalisation du travail, caractérisées par le sentiment d'urgence et les difficultés à gérer son temps, sont certainement les principaux facteurs limitatifs, et ce indépendamment du degré de management des performances en vigueur dans le service. En effet, non seulement l'ensemble des services analysés vit un processus de transformation plus ou moins avancé, mais encore il existe d'autres facteurs de pression à la performance que les formes de management des performances que nous avons considérées (notamment les attentes des usagers qui deviennent de plus en plus fortes et complexes et la gestion plus générale de l'organisation, marquée par l'austérité actuelle (Emery et Wyser, à paraître); dans ce contexte, la disponibilité et la flexibilité du personnel (notamment la gestion de leur temps) s'en trouvent fort réduites, ce qui limite l'accès à la FC ;

- la reconnaissance et la légitimité de la formation continue demeurent l'une comme l'autre assez faibles dans les faits ; il faut une motivation personnelle forte pour ne pas se décourager face au manque de soutien vis-à-vis de toute formation qui va au-delà de la fonction ou le domaine d'activités actuels, de même que face à la faible reconnaissance et valorisation qui suivent une action de formation continue ;

- en fonction du pouvoir qui lui est accordé par les modes de délégation actuels, la hiérarchie de proximité peut contrecarrer les projets et initiatives de FC des acteurs, voire bloquer la circulation ascendante de l'information vers le sommet de la hiérarchie ;

- la proximité de la retraite et l'ancienneté constituent souvent des facteurs de désengagement en matière de $\mathrm{FC}$, un comportement assez fréquent de la part de ces acteurs, pour lesquels la pratique de la FC n'est jamais devenue un acte naturel ;

- le sous-développement des pratiques de gestion des ressources humaines, et plus particulièrement de gestion des compétences, n'offre guère de cadre référentiel permettant d'ancrer les actions de FC dans une logique de développement qui pourrait servir autant les intérêts de l'employeur que ceux de l'employé ; des outils tels que les cartes des emplois, l'évaluation des profils de compétences et les filières d'évolution professionnelles sont embryonnaires ou en voie d'élaboration.

Il résulte de ces atouts et contraintes une marge de manœuvre plutôt restreinte, mais différente dans le domaine de spécialisation et en dehors de ce domaine. Étant donné l'importance accrue accordée à la dimension technique du travail, la marge de 
manœuvre à l'intérieur du domaine de spécialisation est théoriquement importante; en pratique toutefois, elle est plus faible, car délimitée par le rythme et la prégnance des changements en cours, par les ressources limitées, le manque de temps et les freins de la hiérarchie de proximité. Pour autant, les personnes rencontrées se déclarent satisfaites de cette situation et n'en perçoivent plus les aspects restrictifs et le potentiel de marge de manœuvre qui demeure inexploré ; cette forme d'autolimitation par inertie naturelle (" on se laisse aller ») explique également la distance séparant marge de manœuvre théorique et réelle. En dehors du domaine de spécialisation, la marge de manœuvre est généralement faible, étant donné le manque de soutien et la difficulté de se légitimer, mais elle s'accroît notablement lorsqu'il existe un système de quota annuel de jours de FC que l'on peut choisir. De tels quotas sont prévus par certains statuts du personnel, qui octroient un droit annuel à la formation de X jours par an.

Les stratégies-types identifiées dans nos unités d'analyse représentent des logiques générales qui sous-tendent les comportements de FC concrètement relevés. Elles peuvent être distinguées en fonction de l'impulsion qui est à l'origine des comportements de FC.

Lorsque l'initiative individuelle prédomine, les stratégies s'étendent :

- d'une forme «d'hygiène professionnelle », semiconsciente, par laquelle les personnes interrogées estiment que la FC fait peu ou prou partie intégrante de leur travail ;

- en passant par une stratégie définissant la FC comme un "levier d'amélioration professionnel et personnel », conçu dans l'optique de correspondre au mieux aux exigences de son travail et non pas pour améliorer son employabilité à long terme ;

- jusqu'à une stratégie de type "self-service», marquée par un individualisme nourri de la vision personnelle que l'acteur se fait de son travail et de son évolution, qui se retrouve d'ailleurs dans les services ne disposant pas d'un MP intensif.

Lorsque l'impulsion de la direction domine, situation où la véritable dimension stratégique de l'acteur paraît moins évidente, on trouve :
- une stratégie où les personnes perçoivent la FC comme un "outil de changement " permettant à la direction de faire passer ses nouvelles exigences ;

- jusqu'à une stratégie "résignée-passive », qui pousse les personnes à entreprendre des actions de formation continue uniquement sur leur temps privé (ou hors temps de travail), par exemple en s'inscrivant à titre privé dans une école pour adultes (typiquement : inscription à un cours de langue dispensé par un institut privé).

Parmi les points communs identifiés entre ces stratégies-types, relevons l'orientation avant tout technique et fonctionnelle, dans une perspective à court ou moyen terme, ayant recours à des ressources formationnelles internes essentiellement.

Ainsi l'analyse qualitative nous a-t-elle permis d'approfondir les constats faits dans la partie quantitative, brièvement résumée ci-dessus, tout en confirmant les tendances relevées, à savoir l'intensification des formations continues imposées dans un environnement soumis au management des performances. Il est notoire que les pratiques de management des performances participent d'un mouvement plus général porté vers la rationalisation des moyens à disposition et dont l'effet sur les comportements de FC n'est pas univoque: dans certains cas, il peut désankyloser les services et provoquer une dynamique favorable à la formation continue, mais poussé à l'extrême, il entraînera une mécanisation des processus et rapports sociaux internes à l'organisation. D'une manière plus générale, nous observons ce que nous pourrions appeler un «effet service public », puisque d'une part, malgré la forte pression générale, le total des heures de formation continue a tendance à être plus élevé que celui issu des analyses des enquêtes suisses sur la population active effectuées par l'Office fédéral de la statistique (OFS) et que, d'autre part, le soutien organisationnel à ces actions de formation continue demeure important.

La reconstitution des stratégies en matière de formation continue s'est révélée pour nous à la fois passionnante et déroutante : passionnante parce qu'elle nous 
obligeait à considérer la logique d'ensemble de l'activité professionnelle des personnes rencontrées, voire même de l'ensemble de leurs activités; déroutante parce qu'il aurait fallu pouvoir discuter plus longuement avec elles, vivre une partie de leur fonction pour prétendre réellement décrypter leur « réalité », dans une approche quasi clinique de reconstitution des comportements individuels. Ce que notre méthodologie (et nos ressources !) ne permettait guère.

Au-delà des modes d'actions individuels, il apparaît que le personnel des organisations publique vit une transition marquante que l'on pourrait résumer ainsi : le passage d'un système où la carrière se développait surtout à l'ancienneté, sans réelle formation continue structurée, à un système fortement structuré mais qui ne garantit plus la progression de carrière, ni même, à l'heure actuelle tout au moins, la reconnaissance des formations continues suivies. Une situation qui produit une forme de vide permettant l'émergence d'un paradoxe au sein de la formation continue : en l'absence d'un nouveau cadre de progression professionnelle, au moins sous forme de cartes d'emploi, il s'agit de résoudre le défi de la « responsabilisation personnelle en matière de formation continue", exigence présente maintenant dans la plupart des politiques du personnel (un discours de plus en plus présent aussi au niveau international), sous la prégnance d'un processus formationnel de plus en plus imposé par une logique collective de développement des compétences, pilotée au niveau de l'unité organisationnelle (service), voire de l'ensemble de l'organisation selon sa taille. D'autre part, ce défi doit être relevé alors que l'on observe la tendance à dégager sur la sphère privée toute démarche formationnelle plus large et à plus long terme, pour laquelle le soutien organisationnel est faible, voire inexistant.

Cette situation de vide, souhaitons-le passagère, se traduit par l'absence complète de projets professionnels de la part de nos interlocuteurs, tout occupés à intégrer le changement qui les touche dans leur poste actuel. L'absence de lisibilité des conséquences associées à un investissement personnel dans la formation continue, un phénomène que l'on retrouve dans d'autres études (Von Berdeleben, Beicht, Herget et Krekel, 1996), ne contribue naturellement pas à stimuler les personnes dans ce sens. Si des études à plus large spectre, comme celle de Buchmann (1999), mettent en évidence des liens entre formation continue et amélioration des possibilités de promotion et chances d'évolution professionnelle individuelle, c'est un élément qui ne ressort que de manière très indirecte dans le discours de nos interlocuteurs, presque une question de foi plus que d'éléments concrets; bien entendu, l'absence de dimension longitudinale de notre étude ne permettait pas d'analyser à plus long terme le «retour sur investissement » de la formation continue suivie.

$\mathrm{Au}$ moment où la littérature spécialisée exhorte les managers et les collaborateurs à prendre en main leur avenir professionnel, et donc, en partie au moins, leur formation continue, les tendances ressortant de nos entretiens montrent le rôle absolument prédominant que joue l'adaptation à l'emploi actuel et aux objectifs immédiatement opérationnels, même dans les services sans management des performances. Elles confirment aussi les nombreuses études antérieures montrant que la marge de manœuvre et le recours à la formation continue dépendent directement du niveau de formation initial (Bodart-Senn et Schräder-Naef, 2000). On peut se demander alors si l'exigence d'anticipation prônée par la gestion des ressources humaines (Emery, 2003), et la formation en particulier (voir par exemple Meignant, 2002), ne relève pas, en l'état actuel, de vœux pieux très difficiles à concrétiser, si ce n'est bien sûr en s'investissant en dehors du temps de travail; ce que certaines personnes rencontrées n'hésitent pas à faire, en développant différents comportements d'autoformation, un domaine longtemps délaissé par la gestion, et la gestion des ressources humaines en particulier, mais en voie de reconnaissance et d'appui (Moisan, 2002). En considérant les logiques formationnelles globales (touchant l'ensemble du personnel) mises en exergue dans les différents services analysés, certaines conclusions plus générales peuvent être formulées.

Tout d'abord, relevons une ouverture de principe des directions à la formation continue, parfois avec des ressources formationnelles (budgétaires) nettement accrues, mais qui demeure fréquemment une ouverture passive, relayée par le message de la « responsabilisation personnelle en matière de formation continue », les personnes interrogées devant prendre l'initiative et braver le critère de l'utilité fonctionnelle 
à court terme. Il semble ainsi urgent de repenser le «contrat psychologique $»^{4}$, au sens de Cappelli (1999), existant entre employés et employeurs publics aujourd'hui. Ce nouveau contrat psychologique dans les services publics devrait inclure, aux côtés des attentes relationnelles (dont les ancrages sont à redéfinir) et transactionnelles fortement mises en exergue par le management des performances, des attentes formationnelles, comme le révèlent les recherches récentes en la matière (Lemire et Rouillard, 2001 ; Lemire et Rouillard, 2005).

Une importante formation auto-générée par le changement est à relever, fonctionnelle et à court terme, et en bonne partie obligatoire : elle est nécessaire pour acquérir les nouvelles compétences, exercer les nouveaux comportements d'encadrement; une formation spécifique à l'organisation et/ou au service et qui, paradoxalement car elle ne tient pas compte des besoins individuels, augmente l'employabilité des personnes concernées, sans qu'elles en aient directement conscience d'ailleurs ${ }^{5}$. Mais qui produit également une forme d'injonction paradoxale que nous pouvons résumer ainsi : «prends en main ta formation continue, nous (la direction) allons la maitriser de plus en plus ».

Notons également une structuration progressive de la colonne vertébrale technique au sein des différents services analysés, c'est-à-dire que l'accès aux métiers devient réglementé et nécessite la réussite de modules de formation spécifiques; cette structuration a pour corollaire une forme de dépossession du micro-management des échanges traditionnels entre collègues (forme de compagnonnage), une inversion $\mathrm{du}$ sens du processus formationnel (du haut vers le bas plutôt que du bas vers le haut), et l'embryon de ce qui pourrait aboutir à une forme de management des compétences, voire de management des connais-

\footnotetext{
${ }^{4}$ De manière résumée, le contrat psychologique représente l'ensemble des attentes, informelles et donc non documentées $a$ priori, qui se développent entre les employeurs et leur personnel. Ces attentes - relatives aux conditions d'emploi, à la manière d'être encadré... et en particulier d'être formé - se révèlent très importantes pour comprendre la motivation au travail et l'implication du personnel dans l'organisation. Notion développée initialement dans les années 60, notamment par Edgar Schein.

${ }^{5}$ Il s'agit de la maitrise de nouvelles technologies ou de nouvelles approches de gestion, par exemple, nécessaires à l'introduction de la NGP.
}

sances au sens de Bück (1999), c'est-à-dire une exploitation systématique des connaissances acquises dans le cadre de la réalisation du travail, non seulement pour qualifier les acteurs et accroître leurs compétences individuelles, mais également pour développer le capital connaissances de l'organisation elle-même, par exemple sous la forme de systèmes experts. Mais les outils mis en place demeurent trop rares: un fossé sépare la théorie des applications pratiques, dans les services publics tout au moins, qui restent pauvres en la matière. En effet, aucun des services analysés ne recourt à une forme de management des compétences ou des connaissances, puisque la seule tendance constatée est l'orchestration progressive, par la direction, de la formation technique propre au service. La gestion des compétences acquises est toujours réalisée sur une base intuitive en tenant compte de l'ancienneté et de l'expérience acquise dans la fonction. Mais cette forme de prise en compte devient manifestement insuffisante si l'on considère l'engagement multi-facettes/multicontextes qui, demain, attend les cadres et le personnel des organisations publiques. Il s'agit donc d'aller au-delà du carnet-formation, inventaire de base des formations suivies, un outil très simple mais dont l'utilisation n'est pas encore généralisée, pour imaginer des systèmes de validation des compétences acquises, quel que soit le mode d'acquisition (classique, expérientiel, par auto-formation) (Thom et Harasymowicz-Birnbach, 2003).

Ainsi, s'ils ne sont pas pris en considération sous une forme ou une autre, les échanges de type compagnonnage, très souvent mentionnés encore aujourd'hui comme source formationnelle, y compris pour les cadres, sont à terme menacés par cette structuration progressive de la FC par le centre. Cette prise en compte devrait notamment aboutir à mieux définir, et valoriser, les anciens et les nouveaux rôles formationnels : les anciens parce que le management des performances aura tendance à terme, nous pouvons le mettre en hypothèse, à focaliser le personnel sur la production plutôt que sur l'échange avec les collègues, provoquant une forme d'égoïsme individuel fondamentalement néfaste à la transmission des savoir-faire (Knoepfel 2002) ; d'autre part, par une meilleure reconnaissance de celles et ceux qui assument des rôles formationnels dûment identifiés 
(animation de cours internes, parrainage, etc.), des formules qui tendent à se développer.

D'une manière plus générale, nous avons constaté que le management de la formation continue est en mutation. Les organisations publiques analysées ne disposent que d'une vue très partielle des actions de formation ayant réellement lieu en leur sein. Et cela n'est pas forcément dû à une forte décentralisation des ressources ou des budgets formationnels, comme c'est le cas dans certaines de nos organisations. Les indicateurs gérés sont insuffisants, les services concernés ne disposant d'informations complètes ni sur les ressources financières consommées, a fortiori s'il s'agit de calculer l'investissement formationnel en coûts complets incorporant le temps passé et les autres ressources consommées, ni sur le nombre total de journées de formation continue pour chaque membre du personnel, et encore moins s'il s'agit d'incorporer d'autres sources formationnelles telles que manifestations externes, foires, etc., confirmant les résultats d'autres recherches (Bach, 2000).

Ainsi, pendant longtemps, l'organisation publique a essentiellement produit, en se souciant peu de la formation continue. Plus récemment, elle s'est mise à produire pour qualifier, s'engageant comme un employeur formateur ${ }^{6}$, puis actuellement, nous pour- rions dire qu'elle qualifie pour produire, puisque la formation est de plus en plus instrumentalisée, conçue comme levier d'un changement dont les effets sont attendus dans le court terme. La difficulté pour nos interlocuteurs d'accéder à une formation continue plus large - hormis dans le système du quota, qui représente une solution organisationnelle ad hoc mais qui, rappelons-le, ne peut s'appuyer sur aucun « droit à la formation continue » dans notre pays - en témoigne. Elle illustre le fossé probablement grandissant entre la formation continue dans les organisations, plus maîtrisée et stratégique, et la formation continue telle qu'appréhendée dans l'éducation des adultes, conçue à plus long terme et moins focalisée.

Reste à inventer de nouvelles alliances et de nouveaux modes d'organisation, permettant de produire et de qualifier, en s'inspirant des innombrables suggestions d'organisations post-hiérarchiques et tayloriennes (Le Menestrel et Schpilberg, 1999), dans la lignée des propositions de Robert et Haspel qui mettent en exergue les nombreuses synergies existant entre ces deux modes (Ropert et Haspel, 1996). Nous avons des raisons d'espérer en considérant les différentes tendances évoquées ci-dessus.

${ }^{6}$ Nous pensons ici à l'initiative sur les entreprises formatrices, auxquelles les administrations publiques ont sensiblement participé, une initiative développée pour relancer les places d'apprentissage en Suisse.

\section{Bibliographie}

Bach P. (2000), Image de la formation dans les entreprises romandes, Lausanne, Rapport de recherche de l'ARFOR.

Bochsler D., Koller C., Sciarini P., Traimond S. et Trippolini I. (2004), Les cantons suisses sous la loupe, Berne, Haupt.
Bodart-Senn J. et Schräder-Naef R. (2000), La formation des adultes en Suisse, OCDE Background report, OCDE, Paris.

Buchmann C. (1999), Weiterbildung und Beschäftigungschancen. Programme national de recherche $(P N R)$ 33, Fonds national pour la recherche scientifique, Zürich, Rüegger. 
Bück J.-Y. (1999), Le management des connaissances, Paris, Les Éditions d'Organisation.

Cappelli P. (1999), The New Deal at Work: Managing the Market-Driven Workforce, Harvard, Harvard Business School Press.

Costa P. T. J. (1996), "Work and personality : Use of the NEO-PI-R in industrial / organisational psychology", Applied Psychology : an International Review 45(3), pp. 225-241.

Crozier M. et Friedberg E. (1977), L'acteur et le système, Paris, Points-Politique.

Emery Y. (Ed.) (2000), L'administration dans tous ses états, Actes du colloque des 11 et 12 février 1999, Lausanne, Presses polytechniques universitaires romandes.

Emery Y. (2003), Renouveler la gestion des ressources humaines, Lausanne, Presses polytechniques et universitaires romandes.

Emery Y. (2006), « Réforme et légitimités du secteur public », in Le service public, Bellanger F., Genève, Schulthess, pp. 133-159.

Emery Y., Budde S. et Chuard Delaly M. (2004), La formation continue dans les services publics, Berne, Peter Lang.

Emery Y. et Giauque D. (2005), Paradoxes de la gestion publique, Paris, L'Harmattan.

Emery Y. et Gonin F. (2006), Dynamiser la gestion des ressources humaines, Lausanne, Presses polytechniques et universitaires romandes.

Emery Y. et Lambelet-Rossi L. (2000), Les politiques du personnel : conception, analyse et recommandations, Chavannes-près-Renens (Suisse), Idheap.

Emery Y. et Ueberhart B. (1999), Tendances dans les statuts du personnel des cantons suisses, Lausanne, Idheap.

Emery Y. et Wyser C. (à paraître), “The swiss federal administration in a context of downsizing: the public servants perception about productivity, motivation and ethical issues", Ethics and Integrity of governance: perspectives accross frontiers, Huberts L., Edward Elgar Publishing Ltd.

Friedberg E. (1997), Le pouvoir et la règle : dynamiques de l'action organisée, Paris, Seuil.

Germann R. E. (1996), Administration publique en Suisse, Berne, Haupt.

Knoepfel P. (2002), L'éclatement de l'État - acht Thesen zur Abdankung der Einheitlichen, allgemeinen Staatsverwaltung, Chavannes-près-Renens, Idheap.

Le Menestrel J. et Schpilberg M. (1999), Au revoir et merci, Monsieur Taylor, Paris, Éditions d'Organisation.

Lemire L. et Rouillard C. (2001), Le contrat psychologique: analyse comparée des secteurs public et privé, Montréal, ENAP.

Lemire L. et Rouillard C. (2005), “An empirical exploration of psychological contract violation and individual behaviour: The case of Canadian federal civil servants in Quebec", Journal of Managerial Psychology 20(2), pp. 150-163.

Meignant A. (2002), « Le savoir et l'entreprise, un nouveau paradigme », Organisations et systèmes de formation, Garant M. et Scieur Ph., Bruxelles, De Boeck.

Moisan A. (2002), «Autoformation et ressources humaines ", Personnel, revue ANDCP(430), pp. 2125.

Ropert G. et Haspel R. (1996), Construire des organisations qualifiantes ou comment concilier compétitivité et solidarité, Paris, Éditions d'Organisation.

Thom N. et Harasymowicz-Birnbach J. (Eds) (2003), Wissensmanagement im privaten und öffentlichen Sektor, Zürich, vdf.

Von Berdeleben R., Beicht U., Herget H. et Krekel E. M. (1996), Individuelle Kosten und Nutzen beruflicher Weiterbildung, Berichte zur beruflichen Bildung, Heft 201, Bielefeld, Bundesinstitut für Berufsbildung. 


\section{Résumé}

\section{Formation continue dans les services publics en suisse : quelles stratégies des acteurs dans un environnement managérialisé ?

\author{
Yves Emery
}

Dans l'environnement actuel fortement évolutif, les questions relatives à la formation continue sont plus que jamais essentielles. Nombre de publications les abordent toutefois uniquement sous un angle prescriptif, sans considérer le comportement réel du personnel en matière de formation continue. La recherche dont il est question dans cet article vise à mettre en évidence les stratégies que les agents publics suisses mettent en œuvre, au sein d'organisations soumises à une managérialisation croissante, pour atteindre leurs propres objectifs de formation. Sur la base des analyses effectuées, la conclusion esquisse certains enjeux plus fondamentaux relatifs à l'avenir de la formation continue dans les services publics suisses, enjeux à notre sens largement transposables au sein d'autres pays de l'OCDE (Organisation de coopération et de développement économiques).

\section{Mots clés}

Formation professionnelle en entreprise, Secteur public, Pratique de GRH, Suisse Journal of Economic Literature: M. 53 Training ; H 83 Public Administration 


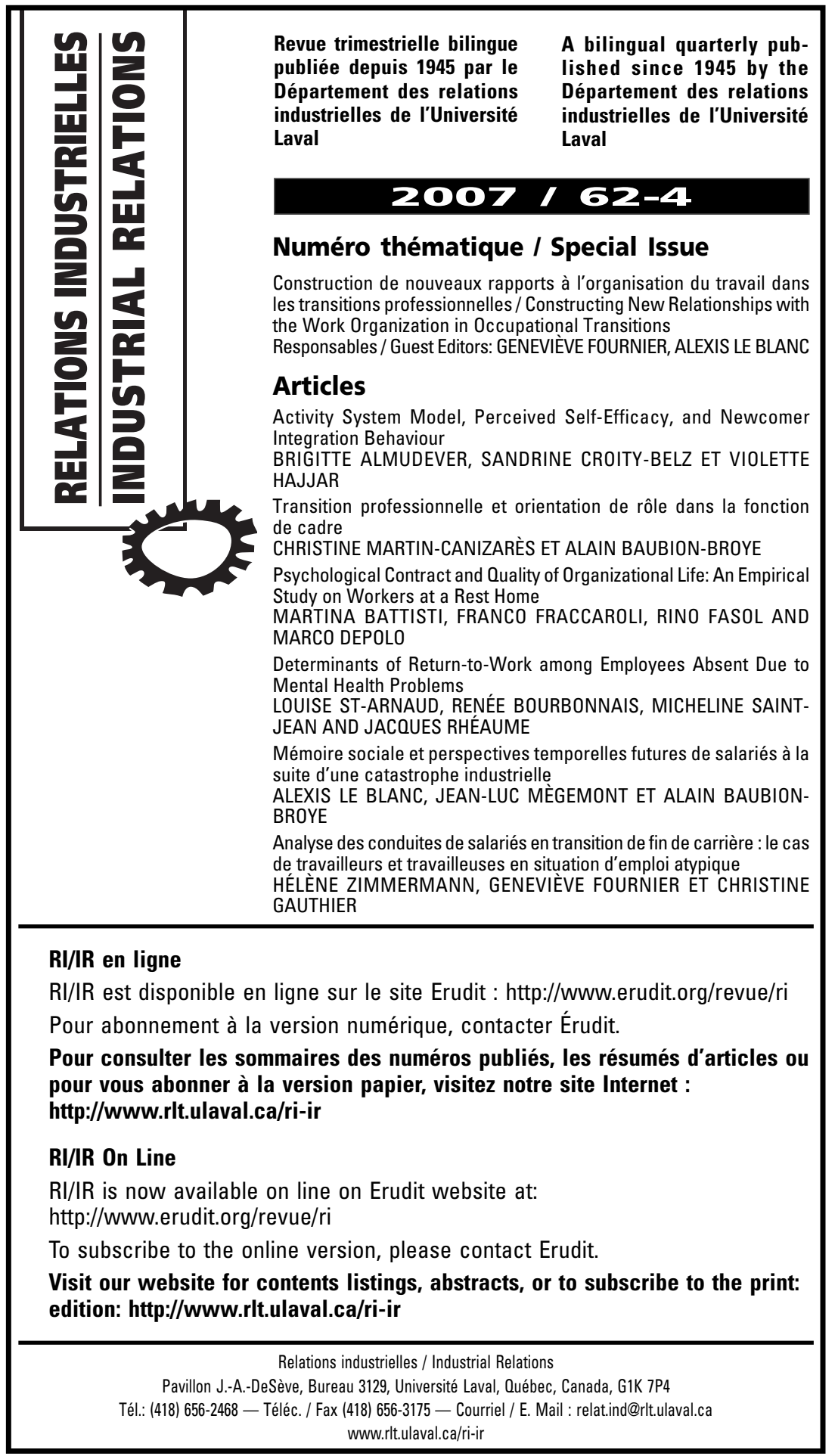

